\title{
FILTRATION OF LINEAR FUNCTIONALS OF PERIODICALLY CORRELATED SEQUENCES
}

UDC 519.21

\section{I. DUBOVETS'KA AND M. P. MOKLYACHUK}

Abstract. The problem of the optimal estimation is considered for the linear functional

$$
A \zeta=\sum_{j=0}^{\infty} a(j) \zeta(-j)
$$

that depends on unknown values of a periodically correlated stochastic sequence $\zeta(j)$; the estimator is constructed from observations of the sequence $\zeta(j)+\theta(j), j \leq 0$, where $\theta(j)$ is a periodically correlated noise. We obtain the mean square error and spectral characteristic of the optimal linear estimate of the functional $A \zeta$ in the case where the spectral densities of the sequences that generate $\zeta(j)$ and $\theta(j)$ are known. For the case where these spectral densities are unknown but a set of admissible spectral densities is given, we find the least favorable spectral density and minimax spectral characteristic for the optimal estimate of the functional $A \zeta$.

\section{INTRODUCTION}

Gladyshev [5 studied spectral properties and representations of periodically correlated sequences by using a relationship between periodically correlated and stationary vector sequences. According to Gladyshev's results, the problem of estimation of periodically correlated sequences is reduced to the corresponding problem for stationary vector sequences. Basic results concerning the representations of periodically correlated sequences in terms of simpler random sequences can be found in the book by Hurd and Miamee [7] and in the papers by Makagon [9, 10.

The classical methods for solving the problems of extrapolation, interpolation, and filtration for stationary processes are developed by Kolmogorov [8], Wiener [18, and Yaglom [19, 20, for the case where the spectral densities are known. The problem of prediction of stationary vector sequences is studied by Rozanov [17. If the spectral densities are not known but a set of admissible spectral densities is given, then the minimax method is used for the problems of estimation. The minimax method is to minimize the error of estimation for all densities of the given class. Grenander [6] is the first who applied the minimax method to the problems of extrapolation for stationary processes. Franke [2, 3], and Franke and Poor [4] considered the problem of minimax extrapolation and filtration for stationary sequences by using the methods of convex optimization. Moklyachuk [11] 14] and Moklyachuk and Masyutka [15] studied the problems of extrapolation, interpolation, and filtration for stationary processes and sequences.

2010 Mathematics Subject Classification. Primary 60G10, 60G25, 60G35; Secondary 62M20, 93E10, $93 \mathrm{E} 11$.

Key words and phrases. Periodically correlated sequence, robust estimate, mean square error, least favorable spectral density, minimax spectral characteristic. 
In this paper, we study the problem of optimal linear estimation of the linear functional

$$
A \zeta=\sum_{j=0}^{\infty} a(j) \zeta(-j)
$$

that depends on unknown values of a periodically correlated sequence $\zeta(j)$. Known are the observations of the sequence $\zeta(j)+\theta(j)$ with $j \leq 0$, where $\theta(j)$ is a periodically correlated sequence that is uncorrelated with $\zeta(j)$. We derive formulas for the spectral characteristic and mean square error of the estimate for the functional (11) in the case where the spectral densities of the sequence $\zeta(j)$ and noise $\theta(j)$ are known. If the densities are unknown but a set of admissible densities is given, then we present formulas for the calculation of the least favorable density and minimax spectral characteristic of the optimal linear estimate of functional (1).

\section{Periodically correlated sequences GENERATED BY STATIONARY VECTOR SEQUENCES}

Periodically correlated sequences are examples of the so-called stochastic sequences with periodic structure introduced in [7].

Definition 2.1. A sequence of complex-valued random variables $\zeta(n), n \in \mathbb{Z}$, with finite second moment, $\mathrm{E}|\zeta(n)|^{2}<+\infty$, is called periodically correlated with period $T$ if

$$
\begin{gathered}
\mathrm{E} \zeta(n+T)=\mathrm{E} \zeta(n), \\
\mathrm{E} \zeta(n+T) \overline{\zeta(m+T)}=R(n+T, m+T)=R(n, m),
\end{gathered}
$$

and if there is no number $T^{\prime}$ smaller than $T>0$ for which equalities (2) and (3) with $T^{\prime}$ instead $T$.

The notion of periodically correlated sequences is introduced by Gladyshev in the paper [5]. Bennet [1] uses the term cyclostationary for random periodic processes.

Definition 2.2. A complex-valued $T$-dimensional random sequence

$$
\vec{\xi}(n)=\left\{\xi_{k}(n)\right\}_{k=0}^{T-1}, \quad n \in \mathbb{Z},
$$

with finite second moment $\mathrm{E}\|\vec{\xi}(n)\|^{2}<\infty$, is called stationary if

$$
\begin{gathered}
\mathrm{E} \xi_{k}(n)=m_{k}, \\
\mathrm{E} \xi_{k}(n) \overline{\xi_{j}(m)}=R_{k j}(n, m)=R_{k j}(n-m)
\end{gathered}
$$

for all $n, m \in \mathbb{Z}$ and all $j, k \in\{0,1, \ldots, T-1\}$.

In this case, the matrix

$$
R(n)=\left\{R_{k j}(n)\right\}_{k, j=0}^{T-1}
$$

is called the correlation matrix of the stationary $T$-dimensional sequence $\vec{\xi}(n)$.

Theorem 2.1 (Gladyshev [5]). A sequence $\zeta(n)$ is periodically correlated with period $T$ if and only if there exists a T-dimensional stationary sequence $\vec{\xi}(n)=\left\{\xi_{k}(n)\right\}_{k=0}^{T-1}$ such that $\zeta(n)$ admits the representation

$$
\zeta(n)=\sum_{k=0}^{T-1} e^{2 \pi i n k / T} \xi_{k}(n), \quad n \in \mathbb{Z} .
$$


We say that the sequence $\vec{\xi}(n)$ generates the sequence $\zeta(n)$ or, in other words, $\zeta(n)$ is generated by $\vec{\xi}(n)$.

Denote by $f^{\vec{\xi}}(\lambda)$ the matrix of spectral density of a $T$-dimensional stationary sequence $\vec{\xi}(n)=\left\{\xi_{k}(n)\right\}_{k=0}^{T-1}$. Let $f^{\vec{\zeta}}(\lambda)$ be the matrix of spectral densities of a $T$-dimensional stationary sequence $\vec{\zeta}(n)$ constructed from the periodically correlated sequence $\zeta(n)$ by splitting in the blocks of length $T$. This means that a coordinate $p$ of the vector $\vec{\zeta}(n)$ is equal to

$$
[\vec{\zeta}(n)]^{p}=\zeta(n T+p), \quad n \in \mathbb{Z}, p=0,1, \ldots, T-1 .
$$

If the spectral density $f^{\vec{\xi}}(\lambda)$ exists, then the spectral density $f^{\vec{\zeta}}(\lambda)$ exists, too, and moreover

$$
f^{\vec{\zeta}}(\lambda)=T \cdot V(\lambda) f^{\vec{\xi}}(\lambda / T) V^{-1}(\lambda),
$$

where $V(\lambda)$ is the unitary matrix whose entry $(k, j)$ equals

$$
v_{k j}(\lambda)=\frac{1}{\sqrt{T}} e^{2 \pi i j k / T+i k \lambda / T}, \quad k, j=0,1, \ldots, T-1 .
$$

Since $V(\lambda)$ is continuous for $\lambda \in[-\pi, \pi)$ and the inverse matrix to $V(\lambda)$ exists, we can rewrite relation (5) as

$$
f^{\vec{\xi}}(\lambda)=T^{-1} \cdot V^{-1}(T \lambda) f^{\vec{\zeta}}(T \lambda) V(T \lambda) .
$$

\section{The PRoblem of Filtration. The Classical solution}

Let $\zeta(n)$ and $\theta(n)$ be two uncorrelated periodically correlated random sequences with period $T$. Consider the problem of the optimal linear estimation of the functional

$$
A \zeta=\sum_{j=0}^{\infty} a(j) \zeta(-j)
$$

that depends on unknown values $\zeta(n)$. We estimate $A \zeta$ from observations $\zeta(j)+\theta(j)$ for $j \leq 0$. This problem is called the problem of linear filtration.

Let $\vec{\xi}(n)=\left\{\xi_{k}(n)\right\}_{k=0}^{T-1}$ and $\vec{\eta}(n)=\left\{\eta_{k}(n)\right\}_{k=0}^{T-1}$ be two uncorrelated $T$-dimensional stationary random sequences with period $T$ generating the periodically correlated random sequences $\zeta(n)$ and $\theta(n)$, respectively. The $T$-dimensional stationary sequences $\vec{\zeta}(n)$ and $\vec{\theta}(n)$ are random sequences obtained by splitting the periodically correlated sequences $\zeta(n)$ and $\theta(n)$ in the blocks of length $T$.

The relationships between the matrices of spectral densities

$$
f^{\vec{\xi}}(\lambda)=\left\{f_{k j}^{\vec{\xi}}(\lambda)\right\}_{k, j=0}^{T-1}, \quad f^{\vec{\eta}}(\lambda)=\left\{f_{k j}^{\vec{\eta}}(\lambda)\right\}_{k, j=0}^{T-1}
$$

of $T$-dimensional stationary sequences $\vec{\xi}(n)$ and $\vec{\eta}(n)$ and matrices of spectral densities $f^{\vec{\zeta}}(\lambda)$ and $f^{\vec{\theta}}(\lambda)$ of the stationary vector sequences $\vec{\zeta}(n)$ and $\vec{\theta}(n)$ are given by

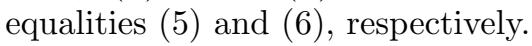

Using relationship (4) between the periodically correlated sequences and stationary vector sequences, we rewrite the functional $A \zeta$ as

$$
\begin{aligned}
A \zeta & =\sum_{j=0}^{\infty} a(j) \zeta(-j)=\sum_{j=0}^{\infty} a(j) \sum_{k=0}^{T-1} e^{-2 \pi i j k / T} \xi_{k}(-j)=\sum_{j=0}^{\infty} \sum_{k=0}^{T-1} a(j) e^{-2 \pi i j k / T} \xi_{k}(-j) \\
& =\sum_{j=0}^{\infty} \vec{a}^{\top}(j) \vec{\xi}(-j)=A \vec{\xi},
\end{aligned}
$$

where $\vec{a}(j)=\left(a_{0}(j), \ldots, a_{T-1}(j)\right)^{\top}$ and $a_{k}(j)=a(j) e^{-2 \pi i j k / T}$ for $k=0,1, \ldots, T-1$. 
Consider the problem of the optimal linear estimation of the functional

$$
A \vec{\xi}=\sum_{j=0}^{\infty} \vec{a}^{\top}(j) \vec{\xi}(-j)
$$

that depends on unknown values $\vec{\xi}(j)$. We estimate the functional $A \vec{\xi}$ from observations $\vec{\xi}(j)+\vec{\eta}(j)$ for $j \leq 0$.

Assume that the sequence of coefficients

$$
\vec{a}(j)=\left\{a_{k}(j)\right\}_{k=0}^{T-1}=\left\{a(j) e^{-2 \pi i j k / T}\right\}_{k=0}^{T-1}, \quad j \geq 0,
$$

defining the functional $A \vec{\xi}=A \zeta$ is such that

(7) $\sum_{j=0}^{\infty} \sum_{k=0}^{T-1}\left|a_{k}(j)\right|=T \sum_{j=0}^{\infty}|a(j)|<\infty, \quad \sum_{j=0}^{\infty}(j+1)\|\vec{a}(j)\|^{2}=T \sum_{j=0}^{\infty}(j+1)|a(j)|^{2}<\infty$.

Note that the second moment of the functional $A \vec{\xi}=A \zeta$ is finite under condition (7).

Let a stationary sequence $\vec{\xi}(j)+\vec{\eta}(j)$ admit the canonical moving average representation given by

$$
\vec{\xi}(j)+\vec{\eta}(j)=\sum_{u=-\infty}^{j} d(j-u) \vec{\varepsilon}(u)
$$

where $d(k)=\left\{d_{i j}(k)\right\}_{i=0, \ldots, T-1}^{j=0, \ldots, m-1}$ and where $\vec{\varepsilon}(u)=\left\{\varepsilon_{k}(u)\right\}_{k=0}^{m-1}$ is a stationary vector white noise sequence, that is,

$$
\mathrm{E} \varepsilon_{k}(n) \overline{\varepsilon_{j}(m)}=\delta_{k j} \delta_{n m} .
$$

Here and in what follows $\delta_{k l}$ denotes the Kronecker symbol, namely $\delta_{k k}=1$ and $\delta_{k l}=0$ for $k \neq l$.

Then the matrix of spectral densities of the sequence $\vec{\xi}(j)+\vec{\eta}(j)$ is of the form

$$
f^{\vec{\xi}}(\lambda)+f^{\vec{\eta}}(\lambda)=T^{-1} V^{-1}(T \lambda)\left(f^{\vec{\zeta}}(T \lambda)+f^{\vec{\theta}}(T \lambda)\right) V(T \lambda)
$$

according to relation (6). Moreover the matrix admits the canonical factorization

$$
\text { (8) } T^{-1} V^{-1}(T \lambda)\left(f^{\vec{\zeta}}(T \lambda)+f^{\vec{\theta}}(T \lambda)\right) V(T \lambda)=d(\lambda) d^{*}(\lambda), \quad d(\lambda)=\sum_{k=0}^{\infty} d(k) e^{-i k \lambda},
$$

where $d^{*}(\lambda)=\overline{d(\lambda)}^{\top}$ is the matrix conjugate to $d(\lambda)$.

Let either the spectral density $T^{-1} V^{-1}(T \lambda) f^{\vec{\zeta}}(T \lambda) V(T \lambda)$ admit the canonical factorization

$$
T^{-1} V^{-1}(T \lambda) f^{\vec{\zeta}}(T \lambda) V(T \lambda)=\varphi(\lambda) \varphi^{*}(\lambda), \quad \varphi(\lambda)=\sum_{k=0}^{\infty} \varphi(k) e^{-i k \lambda}
$$

where $\varphi(k)=\left\{\varphi_{i j}(k)\right\}_{i=0, \ldots, T-1}^{j=0, \ldots, m-1}$, or the spectral density $T^{-1} V^{-1}(T \lambda) f^{\vec{\theta}}(T \lambda) V(T \lambda)$ admit the canonical factorization

$$
T^{-1} V^{-1}(T \lambda) f^{\vec{\theta}}(T \lambda) V(T \lambda)=\psi(\lambda) \psi^{*}(\lambda), \quad \psi(\lambda)=\sum_{k=0}^{\infty} \psi(k) e^{-i k \lambda},
$$

where $\psi(k)=\left\{\psi_{i j}(k)\right\}_{i=0, \ldots, T-1}^{j=0, \ldots, m-1}$. Then the factorization (8) of the density

$$
T^{-1} V^{-1}(T \lambda)\left(f^{\vec{\zeta}}(T \lambda)+f^{\vec{\theta}}(T \lambda)\right) V(T \lambda)
$$

follows if one of the densities given by (9) or (10) is regular. 
Denote by $L_{2}(f)$ the Hilbert space of vector functions $b(\lambda)=\left\{b_{k}(\lambda)\right\}_{k=0}^{T-1}$ that are integrable with respect to the measure with density $f(\lambda)=\left\{f_{k j}(\lambda)\right\}_{k, j=0}^{T-1}$, namely $b \in$ $L_{2}(f)$ if and only if

$$
\int_{-\pi}^{\pi} b^{\top}(\lambda) f(\lambda) \overline{b(\lambda)} d \lambda=\int_{-\pi}^{\pi} \sum_{k, l=0}^{T-1} b_{k}(\lambda) \overline{b_{l}(\lambda)} f_{k l}(\lambda) d \lambda<+\infty .
$$

Denote by $L_{2}^{-}(f)$ the subspace of $L_{2}(f)$ generated by functions of the form

$$
e^{i j \lambda} \delta_{k}, \quad \delta_{k}=\left\{\delta_{k l}\right\}_{l=0}^{T-1}, \quad k=0,1, \ldots, T-1, \quad j \leq 0 .
$$

A linear estimate $\hat{A} \zeta$ of the functional $A \zeta$ constructed from observations $\zeta(j)+\theta(j)$ for $j \leq 0$ is determined by the spectral characteristic

$$
h\left(e^{i \lambda}\right) \in L_{2}^{-}\left(f^{\vec{\zeta}}+f^{\vec{\theta}}\right)
$$

and can be written as

$$
\hat{A} \zeta=\int_{-\pi}^{\pi} h^{\top}\left(e^{i \lambda}\right)\left(Z^{\xi+\eta}(d \lambda)\right)=\int_{-\pi}^{\pi} \sum_{k=0}^{T-1} h_{k}\left(e^{i \lambda}\right)\left(Z_{k}^{\xi+\eta}(d \lambda)\right),
$$

where

$$
Z^{\xi+\eta}(\Delta)=\left\{Z_{k}^{\xi+\eta}(\Delta)\right\}_{k=0}^{T-1}
$$

is the orthogonal random measure of the sum of the sequences $\vec{\xi}(j)$ and $\vec{\eta}(j)$ that generate the sequences $\zeta(j)$ and $\theta(j)$, respectively.

The mean square error of the linear estimate $\hat{A} \zeta$ with the spectral characteristic

$$
h\left(e^{i \lambda}\right)=\sum_{k=0}^{\infty} \vec{h}(k) e^{-i k \lambda}
$$

is calculated as

$$
\begin{aligned}
\Delta\left(h ; f^{\vec{\zeta}}, f^{\vec{\theta}}\right)=\mathrm{E}|A \vec{\zeta}-\hat{A} \vec{\zeta}|^{2} \\
=\frac{1}{2 \pi T} \int_{-\pi}^{\pi} A^{\top}\left(e^{i \lambda}\right) V^{-1}(T \lambda) f^{\vec{\theta}}(T \lambda) V(T \lambda) \overline{A\left(e^{i \lambda}\right)} d \lambda \\
\quad+\frac{1}{2 \pi T} \int_{-\pi}^{\pi}\left[A\left(e^{i \lambda}\right)-h\left(e^{i \lambda}\right)\right]^{\top} \\
\quad \times V^{-1}(T \lambda)\left(f^{\vec{\zeta}}(T \lambda)+f^{\vec{\theta}}(T \lambda)\right) V(T \lambda) \overline{\left[A\left(e^{i \lambda}\right)-h\left(e^{i \lambda}\right)\right]} d \lambda \\
\quad-\frac{1}{2 \pi T} \int_{-\pi}^{\pi}\left[A\left(e^{i \lambda}\right)-h\left(e^{i \lambda}\right)\right]^{\top} V^{-1}(T \lambda) f^{\vec{\theta}}(T \lambda) V(T \lambda) \overline{A\left(e^{i \lambda}\right)} d \lambda \\
\quad-\frac{1}{2 \pi T} \int_{-\pi}^{\pi} A^{\top}\left(e^{i \lambda}\right) V^{-1}(T \lambda) f^{\vec{\theta}}(T \lambda) V(T \lambda) \overline{\left[A\left(e^{i \lambda}\right)-h\left(e^{i \lambda}\right)\right]} d \lambda \\
=\|\Psi a\|^{2}+\|D(a-h)\|^{2}-\langle\Psi(a-h), \Psi a\rangle-\langle\Psi a, \Psi(a-h)\rangle,
\end{aligned}
$$


where

$$
\begin{gathered}
A\left(e^{i \lambda}\right)=\sum_{j=0}^{\infty} \vec{a}(j) e^{-i j \lambda}, \quad\|\Psi a\|^{2}=\sum_{k=0}^{\infty}\left\|(\Psi a)_{k}\right\|^{2}, \quad(\Psi a)_{k}=\sum_{l=0}^{k} \psi^{\top}(k-l) \vec{a}(l), \\
\|D(a-h)\|^{2}=\sum_{k=0}^{\infty}\left\|(D(a-h))_{k}\right\|^{2}, \quad(D(a-h))_{k}=\sum_{l=0}^{k} d^{\top}(k-l)(\vec{a}(l)-\vec{h}(l)), \\
\langle\Psi(a-h), \Psi a\rangle=\overline{\langle\Psi a, \Psi(a-h)\rangle}=\sum_{k=0}^{\infty}\left\langle(\Psi(a-h))_{k},(\Psi a)_{k}\right\rangle .
\end{gathered}
$$

For given densities $f^{\vec{\zeta}}(\lambda)$ and $f^{\vec{\theta}}(\lambda)$, the spectral characteristic $h\left(f^{\vec{\zeta}}, f^{\vec{\theta}}\right)$ of the optimal linear estimate $\hat{A} \zeta$ minimizes the mean square error

$$
\begin{aligned}
\Delta\left(f^{\vec{\zeta}}, f^{\vec{\theta}}\right) & =\Delta\left(h\left(f^{\vec{\zeta}}, f^{\vec{\theta}}\right) ; f^{\vec{\zeta}}, f^{\vec{\theta}}\right)=\min _{h \in L_{2}^{-}\left(f^{\vec{\zeta}}+f^{\vec{\theta}}\right)} \Delta\left(h ; f^{\vec{\zeta}}, f^{\vec{\theta}}\right) \\
& =\min _{\hat{A} \vec{\zeta}} \mathrm{E}|A \vec{\zeta}-\hat{A} \vec{\zeta}|^{2} .
\end{aligned}
$$

Let the density of an observable stationary sequence and that of the estimated sequence admit factorizations (8) and (9), respectively. Then the spectral characteristic $h\left(f^{\vec{\zeta}}, f^{\vec{\theta}}\right)$, which is a solution of problem (12), and the mean square error $\Delta\left(f^{\vec{\zeta}}, f^{\vec{\theta}}\right)$ of the optimal estimate $\hat{A} \zeta$, are calculated as

$$
\begin{gathered}
h\left(f^{\vec{\zeta}}, f^{\vec{\theta}}\right)=b^{\top}(\lambda) r^{\vec{\zeta}}\left(e^{i \lambda}\right), \\
\Delta\left(f^{\vec{\zeta}}, f^{\vec{\theta}}\right)=\left\langle c^{\vec{\zeta}}, a\right\rangle-\left\|C^{\vec{\zeta}} \vec{b}\right\|^{2},
\end{gathered}
$$

where

$$
\begin{gathered}
b(\lambda)=\sum_{k=0}^{\infty} b(k) e^{-i k \lambda}, \quad b(\lambda) d(\lambda)=I_{m}, \\
r^{\vec{\zeta}}\left(e^{i \lambda}\right)=\sum_{k=0}^{\infty}\left(C^{\vec{\zeta} \bar{b}}\right)_{k} e^{-i k \lambda}, \quad\left(C^{\vec{\zeta} \bar{b}}\right)_{k}=\sum_{l=0}^{\infty} \overline{b(l)} \vec{c}(l+k), \\
c^{\vec{\zeta}}(k)=\sum_{l=0}^{\infty} \overline{\varphi(l)}(\Phi a)_{l+k}, \\
(\Phi a)_{k}=\sum_{l=0}^{k} \varphi^{\top}(k-l) \vec{a}(l), \quad\left\langle c^{\vec{\zeta}}, a\right\rangle=\sum_{k=0}^{\infty}\left\langle c^{\vec{\zeta}}(k), \vec{a}(k)\right\rangle \\
\left\|C^{\vec{\zeta}} \bar{b}\right\|^{2}=\sum_{k=0}^{\infty}\left\|\left(C^{\vec{\zeta}} \bar{b}\right)_{k}\right\|^{2}
\end{gathered}
$$

If the density of the observable sequence and that of the noise admit factorizations (8) and (10), respectively, then spectral characteristic $h\left(f^{\vec{\zeta}}, f^{\vec{\theta}}\right)$ and the mean square error $\Delta\left(f^{\vec{\zeta}}, f^{\vec{\theta}}\right)$ of the optimal estimate $\hat{A} \zeta$ are given by

$$
\begin{gathered}
h\left(f^{\vec{\zeta}}, f^{\vec{\theta}}\right)=A\left(e^{i \lambda}\right)-b^{\top}(\lambda) r^{\vec{\theta}}\left(e^{i \lambda}\right), \\
\Delta\left(f^{\vec{\zeta}}, f^{\vec{\theta}}\right)=\left\langle c^{\vec{\theta}}, a\right\rangle-\left\|C^{\vec{\theta}} \bar{b}\right\|^{2},
\end{gathered}
$$


respectively, where

$$
\begin{gathered}
r^{\vec{\theta}}\left(e^{i \lambda}\right)=\sum_{k=0}^{\infty}\left(C^{\vec{\theta} \bar{b}}\right)_{k} e^{-i k \lambda}, \quad\left(C^{\vec{\theta}} \bar{b}\right)_{k}=\sum_{l=0}^{\infty} \overline{b(l)} c^{\vec{\theta}}(l+k), \\
c^{\vec{\theta}}(k)=\sum_{l=0}^{\infty} \overline{\psi(l)}(\Psi a)_{l+k}, \\
(\Psi a)_{k}=\sum_{l=0}^{k} \psi^{\top}(k-l) \vec{a}(l), \quad\left\langle c^{\vec{\theta}}, a\right\rangle=\sum_{k=0}^{\infty}\left\langle c^{\vec{\theta}}(k), \vec{a}(k)\right\rangle, \\
\left\|C^{\vec{\theta}} \bar{b}\right\|^{2}=\sum_{k=0}^{\infty}\left\|\left(C^{\vec{\theta}} \bar{b}\right)_{k}\right\|^{2} .
\end{gathered}
$$

Therefore the following result holds.

Theorem 3.1. Let $\zeta(j)$ and $\theta(j)$ be two uncorrelated periodically correlated random sequences with period T. Assume that $f^{\vec{\zeta}}(\lambda)$ and $f^{\vec{\theta}}(\lambda)$ are the spectral density matrices of T-dimensional stationary sequences $\vec{\zeta}(j)$ and $\vec{\theta}(j)$ obtained by splitting the onedimensional periodically correlated sequences $\zeta(j)$ and $\theta(j)$ in the blocks of length $T$, respectively. Assume further that the spectral densities admit canonical factorizations (8) and (9) or (8) and (10). Then the linear optimal estimate of the functional $A \zeta$ constructed from observations $\zeta(j)+\theta(j)$ for $j \leq 0$ is defined by equality (11). The spectral characteristic $h\left(f^{\vec{\zeta}}, f^{\vec{\theta}}\right)$ of this estimate is calculated by formula (13) or (16), respectively. The mean square error $\Delta\left(f^{\vec{\zeta}}, f^{\vec{\theta}}\right)$ is calculated by formula (14) or (17), respectively.

Corollary 3.1. Let $\zeta(j)$ and $\theta(j)$ be two uncorrelated periodically correlated random sequences with period T. Further let $\vec{\zeta}(j)$ and $\vec{\theta}(j)$ be T-dimensional stationary sequences obtained by splitting the one-dimensional periodically correlated sequences $\zeta(j)$ and $\theta(j)$ in blocks of length $T$, respectively. Assume that one of the sequences $\vec{\zeta}(j)$ or $\vec{\theta}(j)$ is a vector white noise sequence whose coordinates have the variance $\sigma^{2}$. Then the spectral characteristic $h\left(f^{\vec{\zeta}}, f^{\vec{\theta}}\right)$ of the optimal linear estimate of the functional $A \zeta$ is calculated by formula (13) or (16). The mean square error of the prediction is equal to

$$
\Delta\left(f^{\vec{\zeta}}, f^{\vec{\theta}}\right)=\sigma^{2}\|\vec{a}\|^{2}-\frac{\sigma^{4}}{T^{2}}\|\bar{b} a\|^{2},
$$

where

$$
\begin{aligned}
\vec{a}=(a(0), a(1), \ldots), \quad\|\vec{a}\|^{2} & =\sum_{k=0}^{\infty}|a(k)|^{2}, \quad\|\bar{b} a\|^{2}=\sum_{k=0}^{\infty}\left\|(\bar{b} a)_{k}\right\|^{2}, \\
(\bar{b} a)_{k} & =\sum_{l=0}^{\infty} \overline{b(l)} \vec{a}(l+k) .
\end{aligned}
$$

Corollary 3.2. If the assumptions of Corollary 3.1 hold, then the mean square error of the optimal linear estimate $a(N) \zeta(-N)$ constructed from the observations $\zeta(j)+\theta(j)$ for $j \leq 0$ is given by

$$
\Delta\left(f^{\vec{\zeta}}, f^{\vec{\theta}}\right)=\sigma^{2}|a(N)|^{2}-\frac{\sigma^{4}}{T^{2}} \sum_{k=0}^{N}\|\overline{b(k)} \vec{a}(N)\|^{2} .
$$

Example 3.1. Consider two uncorrelated two-dimensional stationary sequences

$$
\vec{\xi}(n)=\left(\begin{array}{l}
\xi_{0}(n) \\
\xi_{1}(n)
\end{array}\right) \quad \text { and } \quad \vec{\eta}(n)=\left(\begin{array}{l}
\eta_{0}(n) \\
\eta_{1}(n)
\end{array}\right)
$$


$n \in \mathbb{Z}$, such that $\xi_{0}(n)$ is a one-dimensional stationary Ornstein-Uhlenbeck sequence with spectral density

$$
f_{0}(\lambda)=\frac{5 / 4}{2 \pi \mid 1-1 / 2 \cdot e^{-\left.i \lambda\right|^{2}}}
$$

$\xi_{1}(n)$ is a one-dimensional stationary sequence that is uncorrelated with $\xi_{0}(n)$ whose density is $f_{1}(\lambda)=\frac{3}{2 \pi}\left|1+e^{i \lambda}\right|^{2}, \eta_{0}(n)$ is a white noise sequence with spectral density $g_{0}(\lambda)=\frac{3}{2 \pi}$, and $\eta_{1}(n)$ is a white noise sequence that is uncorrelated with $\eta_{0}(n)$ whose density is $g_{1}(\lambda)=\frac{2}{\pi}$. According to Gladyshev's Theorem 2.1, one can construct two periodically correlated sequences with period $T=2$ such that

$$
\zeta(n)=\xi_{0}(n)+e^{\pi i n} \xi_{1}(n) \text { and } \theta(n)=\eta_{0}(n)+e^{\pi i n} \eta_{1}(n) .
$$

We estimate the linear functional

$$
A \zeta=\alpha \zeta(0)=\alpha \xi_{0}(0)+\alpha \xi_{1}(0)
$$

with $a(0)=\alpha, \alpha \in \mathbb{R}$, and $a(k)=0, k \geqslant 1$.

Evaluating the spectral characteristic $h\left(f^{\vec{\zeta}}, f^{\vec{\theta}}\right)$ by using formula (13), we obtain the estimate

$$
\begin{aligned}
\hat{A} \zeta= & \alpha\left(\frac{3}{2}\right)^{3 / 2}\left(\xi_{0}(0)+\eta_{0}(0)\right)+\frac{\alpha}{3}\left(\xi_{1}(0)+\eta_{1}(0)\right)-\alpha \sqrt{\frac{3}{2}} \frac{1367}{3456}\left(\xi_{0}(-1)+\eta_{0}(-1)\right) \\
& +\alpha \frac{2}{9}\left(\xi_{1}(-1)+\eta_{1}(-1)\right)+\alpha \sqrt{\frac{3}{2}} \frac{1367}{6912}\left(\xi_{0}(-2)+\eta_{0}(-2)\right)-\alpha \frac{2}{9}\left(\xi_{1}(-2)+\eta_{1}(-2)\right) \\
& +2 \alpha \sum_{k=3}^{\infty} \frac{(-1)^{k-1}}{3^{k+1}}\left(\xi_{1}(-k)+\eta_{1}(-k)\right)
\end{aligned}
$$

according to equality (11). The mean square error of this estimate is evaluated from formula (14) as

$$
\Delta\left(f^{\vec{\zeta}}, f^{\vec{\theta}}\right)=\alpha^{2} \cdot 0.596
$$

The spectral density of the two-dimensional stationary sequence $\vec{\zeta}(n)$ is given by (5) and equals

$$
f^{\vec{\zeta}}(\lambda)=\frac{1}{2 \pi}\left(\begin{array}{cc}
\frac{5}{\left|2-e^{-i \lambda / 2}\right|^{2}}+3\left|1+e^{-i \lambda / 2}\right|^{2} & \frac{5 e^{-i \lambda / 2}}{\left|2-e^{-i \lambda / 2}\right|^{2}}-3 e^{-i \lambda / 2}\left|1+e^{-i \lambda / 2}\right|^{2} \\
\frac{5 e^{i \lambda / 2}}{\left|2-e^{-i \lambda / 2}\right|^{2}}-3 e^{i \lambda / 2}\left|1+e^{-i \lambda / 2}\right|^{2} & \frac{5}{\left|2-e^{-i \lambda / 2}\right|^{2}}+3\left|1+e^{-i \lambda / 2}\right|^{2}
\end{array}\right) .
$$

Analogously, the matrix of spectral densities of the two-dimensional stationary sequence $\vec{\theta}(n)$ is equal to

$$
f^{\vec{\theta}}(\lambda)=\frac{1}{2 \pi}\left(\begin{array}{cc}
7 & 3 e^{-i \lambda / 2}-4 e^{-i \lambda / 2} \\
3 e^{i \lambda / 2}-4 e^{i \lambda / 2} & 7
\end{array}\right)
$$

\section{The minimax (Robust) Method of Filtration}

If the matrices of spectral densities $f(\lambda)$ and $g(\lambda)$ are known for two $T$-dimensional stationary sequences $\vec{\zeta}(j)$ and $\vec{\theta}(j)$ obtained by splitting the one-dimensional periodically correlated sequences $\zeta(j)$ and $\theta(j)$ in blocks of length $T$, respectively, then one can use formulas (13)-(17) to evaluate the spectral characteristic and mean square error of the optimal linear estimate of the functional $A \zeta$. If the matrices of densities are not known but a set $D=D_{f} \times D_{g}$ of admissible spectral densities is given, then one can follow the minimax approach to estimate the functional that depends on periodically correlated sequences. We search an estimate that minimizes the mean square error simultaneously for all spectral densities belonging to the class $D$. 
Definition 4.1. Given a set of pairs of spectral densities $D=D_{f} \times D_{g}$, the matrices of spectral densities $f^{0}(\lambda) \in D_{f}$ and $g^{0}(\lambda) \in D_{g}$ are called the least favorable in the class $D$ for the problem of the optimal linear filtration of the functional $A \zeta$ if

$$
\Delta\left(f^{0}, g^{0}\right)=\Delta\left(h\left(f^{0}, g^{0}\right) ; f^{0}, g^{0}\right)=\max _{(f, g) \in D} \Delta(h(f, g) ; f, g) .
$$

Definition 4.2. Given a set of pairs of spectral densities $D=D_{f} \times D_{g}$, the spectral characteristic $h^{0}(\lambda)$ of the optimal linear interpolation of the functional $A \zeta$ is called minimax or robust if

$$
h^{0}(\lambda) \in H_{D}=\bigcap_{(f, g) \in D} L_{2}^{-}(f+g), \quad \min _{h \in H_{D}} \max _{(f, g) \in D} \Delta(h ; f, g)=\max _{(f, g) \in D} \Delta\left(h^{0} ; f, g\right) .
$$

The following results can be checked explicitly by using relations (8)-(17) obtained above (see [15]).

Lemma 4.1. The matrices of spectral densities $f^{0}(\lambda) \in D_{f}$ and $g^{0}(\lambda) \in D_{g}$ that admit the canonical factorizations (8) -(10) are the least favorable in the class $D$ for the problem of the optimal linear filtration of the functional $A \zeta$ if the coefficients in factorizations (8) -(10) determine the solution of the conditional extremum problem

$$
\begin{gathered}
\Delta(f, g)=\left\langle c^{\vec{\zeta}}, a\right\rangle-\left\|C^{\vec{\zeta}} b^{*}\right\|^{2} \rightarrow \sup , \\
f(\lambda)=T V(\lambda) \varphi(\lambda / T)(V(\lambda) \varphi(\lambda / T))^{*} \in D_{f}, \\
g(\lambda)=T V(\lambda)\left(d(\lambda / T) d^{*}(\lambda / T)-\varphi(\lambda / T) \varphi^{*}(\lambda / T)\right) V^{*}(\lambda) \in D_{g},
\end{gathered}
$$

or the conditional extremum problem

$$
\begin{gathered}
\Delta(f, g)=\left\langle c^{\vec{\theta}}, a\right\rangle-\left\|C^{\vec{\theta}} b^{*}\right\|^{2} \rightarrow \sup , \\
g(\lambda)=T V(\lambda) \psi(\lambda / T)(V(\lambda) \psi(\lambda / T))^{*} \in D_{g}, \\
f(\lambda)=T V(\lambda)\left(d(\lambda / T) d^{*}(\lambda / T)-\psi(\lambda / T) \psi^{*}(\lambda / T)\right) V^{*}(\lambda) \in D_{f} .
\end{gathered}
$$

If one of the densities, either (9) or (10), is known, then problems (19) and (20) become conditional extremum problems with respect to the sequence of the coefficients $\{b(k), k \geq 0\}$ of the matrix function $b(\lambda)=\sum_{k=0}^{\infty} b(k) e^{-i k \lambda}$.

Lemma 4.2. Let the spectral density $f(\lambda)$ be known and admit canonical factorization (91). Then the spectral density $g^{0}(\lambda)$ also admits canonical factorizations (8), (10) and is the least favorable density for the optimal linear filtration of the functional $A \zeta$ if

$$
f(\lambda)+g^{0}(\lambda)=T V(\lambda) d^{0}(\lambda / T)\left(V(\lambda) d^{0}(\lambda / T)\right)^{*},
$$

where

$$
d^{0}(\lambda)=\sum_{k=0}^{\infty} d^{0}(k) e^{-i k \lambda}
$$

and where the matrix coefficients $\left\{d^{0}(k), k \geq 0\right\}$ are determined by the solution

$$
\left\{b^{0}(k), k \geq 0\right\}
$$

of the conditional extremum problem

$$
\left\|C^{\vec{\zeta}} \bar{b}\right\|^{2} \rightarrow \inf , \quad g(\lambda)=T V(\lambda) d(\lambda / T)(V(\lambda) d(\lambda / T))^{*}-f(\lambda) \in D_{g} .
$$


Lemma 4.3. Let the spectral density $g(\lambda)$ be known and admit canonical factorization (10). Then the spectral density $f^{0}(\lambda)$ also admits canonical factorizations (8) and (91) and is the least favorable density for the optimal linear filtration of the functional $A \zeta$ if

$$
f^{0}(\lambda)+g(\lambda)=T V(\lambda) d^{0}(\lambda / T)\left(V(\lambda) d^{0}(\lambda / T)\right)^{*}
$$

where

$$
d^{0}(\lambda)=\sum_{k=0}^{\infty} d^{0}(k) e^{-i k \lambda}
$$

and where the matrix coefficients $\left\{d^{0}(k), k \geq 0\right\}$ are determined by the solution

$$
\left\{b^{0}(k), k \geq 0\right\}
$$

of the conditional extremum problem

$$
\left\|C^{\vec{\theta}} \bar{b}\right\|^{2} \rightarrow \inf , \quad f(\lambda)=T V(\lambda) d(\lambda / T)(V(\lambda) d(\lambda / T))^{*}-g(\lambda) \in D_{f} .
$$

The least favorable spectral densities $f^{0}(\lambda) \in D_{f}$ and $g^{0}(\lambda) \in D_{g}$ and the minimax spectral characteristic $h^{0}=h\left(f^{0}, g^{0}\right)$ form a saddle point of the function $\Delta(h ; f, g)$ in the set $H_{D} \times D$. The saddle point inequalities

$$
\Delta\left(h^{0} ; f, g\right) \leq \Delta\left(h^{0} ; f^{0}, g^{0}\right) \leq \Delta\left(h ; f^{0}, g^{0}\right), \quad \forall h \in H_{D}, \forall f \in D_{f}, \forall g \in D_{g},
$$

are satisfied if $h^{0}=h\left(f^{0}, g^{0}\right), h\left(f^{0}, g^{0}\right) \in H_{D}$ and if $\left(f^{0}, g^{0}\right)$ is a solution of the conditional extremum problem

$$
\Delta\left(h\left(f^{0}, g^{0}\right) ; f, g\right) \rightarrow \sup , \quad(f, g) \in D,
$$

where the functional

$$
\begin{aligned}
\Delta\left(h\left(f^{0}, g^{0}\right) ; f, g\right) & \\
= & \frac{1}{2 \pi T} \int_{-\pi}^{\pi}\left(r^{\vec{\theta}, 0}\left(e^{i \lambda}\right)\right)^{\top} b^{0}(\lambda) V^{-1}(T \lambda) f(T \lambda) V(T \lambda)\left(b^{0}(\lambda)\right)^{*} \overline{r^{\vec{\theta}, 0}\left(e^{i \lambda}\right)} d \lambda \\
& +\frac{1}{2 \pi T} \int_{-\pi}^{\pi}\left(r^{\vec{\zeta}, 0}\left(e^{i \lambda}\right)\right)^{\top} b^{0}(\lambda) V^{-1}(T \lambda) g(T \lambda) V(T \lambda)\left(b^{0}(\lambda)\right)^{*} \overline{r^{\vec{\zeta}, 0}\left(e^{i \lambda}\right)} d \lambda
\end{aligned}
$$

depends linearly on unknown densities $f$ and $g$ belonging to the set of admissible densities $D$ and where the functions $r^{\vec{\zeta}, 0}\left(e^{i \lambda}\right)$ and $r^{\vec{\theta}, 0}\left(e^{i \lambda}\right)$ are calculated by relations (15) and (18), respectively, under the conditions that $f^{\vec{\zeta}}(\lambda)=f^{\vec{\zeta}, 0}(\lambda)$ and $f^{\vec{\theta}}(\lambda)=f^{\vec{\theta}, 0}(\lambda)$.

The conditional extremum problem (23) is equivalent to the unconditional extremum problem

$$
\Delta_{D}(f, g)=-\Delta\left(h\left(f^{0}, g^{0}\right) ; f, g\right)+\delta((f, g) \mid D) \rightarrow \inf ,
$$

where $\delta((f, g) \mid D)$ stands for the indicator function of the set $D$. The solution $\left(f^{0}, g^{0}\right)$ of the latter extremum problem is determined by the condition

$$
0 \in \partial \Delta_{D}\left(f^{0}, g^{0}\right)
$$

where $\partial \Delta_{D}\left(f^{0}, g^{0}\right)$ is the subdifferential of the convex functional $\Delta_{D}(f, g)$ at the point $(f, g)=\left(f^{0}, g^{0}\right)$ (see [16] for detail). 


\section{The least favorable SpeCtral Densities in the Class $D_{F, G}$}

Consider the problem of the minimax estimation of the functional $A \zeta$ that depends on periodically correlated sequences $\zeta(j)$ with period $T$ for the set of spectral densities of $T$-dimensional stationary sequences $\vec{\zeta}(j)$ and $\vec{\theta}(j)$ obtained by splitting $\zeta(j)$ and $\theta(j)$ in the blocks of length $T$ :

$$
\begin{aligned}
D_{F, G}=\left\{\left(f^{\vec{\zeta}}(\lambda), f^{\vec{\theta}}(\lambda)\right): \frac{1}{2 \pi T} \int_{-\pi}^{\pi} V^{-1}(T \lambda) f^{\vec{\zeta}}(T \lambda) V(T \lambda) d \lambda=F\right. \\
\\
\left.\quad \frac{1}{2 \pi T} \int_{-\pi}^{\pi} V^{-1}(T \lambda) f^{\vec{\theta}}(T \lambda) V(T \lambda) d \lambda=G\right\}
\end{aligned}
$$

where $F$ and $G$ are specified numerical matrices. Solving the conditional extremum problem (23) with the help of the Lagrange multipliers method, we find that the least favorable spectral densities $f^{\vec{\zeta}, 0}(\lambda)$ and $f^{\vec{\theta}, 0}(\lambda)$ are such that

$$
\begin{aligned}
& \overline{V(T \lambda)}\left(b^{0}(\lambda)\right)^{\top} r^{\vec{\zeta}, 0}(\lambda)\left(r^{\vec{\zeta}, 0}(\lambda)\right)^{*} \overline{b^{0}(\lambda)} V^{\top}(T \lambda)=\vec{\alpha} \vec{\alpha}^{*} \\
& \overline{V(T \lambda)}\left(b^{0}(\lambda)\right)^{\top} r^{\vec{\theta}, 0}(\lambda)\left(r^{\vec{\theta}, 0}(\lambda)\right)^{*} \overline{b^{0}(\lambda)} V^{\top}(T \lambda)=\vec{\beta} \vec{\beta}^{*}
\end{aligned}
$$

Here $\vec{\alpha}=\left(\alpha_{0}, \ldots, \alpha_{T-1}\right)^{\top}$ and $\vec{\beta}=\left(\beta_{0}, \ldots, \beta_{T-1}\right)^{\top}$ denote the Lagrange multipliers. The latter relations hold if

$$
\begin{aligned}
r^{\vec{\zeta}, 0}\left(e^{i \lambda}\right) & =\left(V(T \lambda) d^{0}(\lambda)\right)^{\top} \vec{\alpha} \\
r^{\vec{\theta}, 0}\left(e^{i \lambda}\right) & =\left(V(T \lambda) d^{0}(\lambda)\right)^{\top} \vec{\beta} .
\end{aligned}
$$

Then the least favorable densities are such that

$$
\begin{aligned}
f^{\vec{\zeta}, 0}(\lambda)+f^{\vec{\theta}, 0}(\lambda) & =\vec{\gamma}\left(r^{\vec{\zeta}, 0}\left(e^{i \lambda / T}\right)\right)^{\top} \overline{r^{\vec{\zeta}, 0}\left(e^{i \lambda / T}\right)} \vec{\gamma}^{*} \\
f^{\vec{\zeta}, 0}(\lambda)+f^{\vec{\theta}, 0}(\lambda) & =\vec{\delta}\left(r^{\vec{\theta}, 0}\left(e^{i \lambda / T}\right)\right)^{\top} \overline{r^{\vec{\theta}, 0}\left(e^{i \lambda / T}\right)} \vec{\delta}^{*}
\end{aligned}
$$

where $\vec{\gamma}=\left(\alpha_{0}, \ldots, \gamma_{T-1}\right)^{\top}, \vec{\delta}=\left(\delta_{0}, \ldots, \delta_{T-1}\right)^{\top}$ and $\vec{\gamma} \vec{\alpha}^{\top}=I_{T}, \vec{\delta} \vec{\beta}^{\top}=I_{T}$.

The Lagrange multipliers $\vec{\gamma}$ and $\vec{\delta}$ as well as the matrix coefficients $\left\{b^{0}(k), k \geq 0\right\}$ are determined from the equations of canonical factorizations (8)-(10) for the matrices of spectral densities $f^{\vec{\zeta}, 0}(\lambda)+f^{\vec{\theta}, 0}(\lambda), f^{\vec{\zeta}, 0}(\lambda)$, and $f^{\vec{\theta}, 0}(\lambda)$ and restrictions defining the class $D_{F, G}$.

If one of the spectral densities is known, then one can use one of the relations (25) or (26) to calculate the least favorable spectral densities for a given class $D_{F, G}$. If the matrix of spectral density $f^{\vec{\zeta}}(\lambda)$ is known, then the least favorable spectral density $f^{\vec{\theta}, 0}(\lambda) \in D_{G}$ equals

$$
f^{\vec{\theta}, 0}(\lambda)=\left[\vec{\gamma}\left(r^{\vec{\zeta}}\left(e^{i \lambda / T}\right)\right)^{\top} \overline{r \vec{\zeta}\left(e^{i \lambda / T}\right)} \vec{\gamma}^{*}-f^{\vec{\zeta}}(\lambda)\right]_{+}
$$

If the matrix of spectral density $f^{\vec{\theta}}(\lambda)$ is known, then the least favorable spectral density $f^{\vec{\zeta}, 0}(\lambda) \in D_{F}$ equals

$$
f^{\vec{\zeta}, 0}(\lambda)=\left[\vec{\delta}\left(r^{\vec{\theta}}\left(e^{i \lambda / T}\right)\right)^{\top} \overline{r^{\vec{\theta}}\left(e^{i \lambda / T}\right)} \vec{\delta}^{*}-f^{\vec{\theta}}(\lambda)\right]_{+} .
$$

Therefore the following result holds. 
Theorem 5.1. The least favorable matrices of spectral densities $f^{\vec{\zeta}, 0}(\lambda)$ and $f^{\vec{\theta}, 0}(\lambda)$ in the class $D_{F, G}$ for the problem of the optimal filtration of the functional $A \zeta$ are determined from equations (25) and (26), factorizations (8)-(10), relations (19) and (20), and from restrictions defining the class $D_{F, G}$.

The minimax spectral characteristic $h\left(f^{\vec{\zeta}, 0}, f^{\vec{\theta}, 0}\right)$ of the estimate of $\hat{A} \zeta$ is given by one of the relations (13) or 16).

The mean square error $\Delta\left(f^{\vec{\zeta}, 0}, f^{\vec{\theta}, 0}\right)$ of the optimal filtration is given by one of the relations (14) or (17).

Corollary 5.1. If the matrix of spectral densities $f^{\vec{\zeta}}(\lambda)\left(\right.$ or $\left.f^{\vec{\theta}}(\lambda)\right)$ is known and admits canonical factorization (9) (or (10)), then the least favorable spectral density $f^{\vec{\theta}, 0}(\lambda)$ (or $\left.f^{\vec{\zeta}, 0}(\lambda)\right)$ is determined by relations (27), (21), (8)-(10) (or (28), (22), (8)-(10) ) and restrictions defining the class $D_{F, G}$.

The minimax spectral characteristic $h\left(f^{\vec{\zeta}, 0}, f^{\vec{\theta}, 0}\right)$ of the estimate $\hat{A} \zeta$ is given by one of the relations (13) or (16).

The mean square error $\Delta\left(f^{\vec{\zeta}, 0}, f^{\vec{\theta}, 0}\right)$ of the estimate is given by one of the relations (14) or (17).

Example 5.1. Let $T=1, f^{\vec{\theta}}(\lambda)=\left|1-\sqrt{2} e^{-i \lambda}\right|^{2}$, and $F=5$. We estimate the functional $A \zeta=\kappa \zeta(0), \kappa \in \mathbb{R}$.

We apply formula (28) to evaluate the least favorable spectral density. The coefficients $\{b(0), b(1)\}$ are solutions of the conditional extremum problem (22) written as follows in the case under consideration:

$$
\left\{\begin{array}{l}
(3 b(0)-\sqrt{2} b(1))^{2}+2 b^{2}(0) \rightarrow \min , \\
b^{2}(0)-b^{2}(1)=\frac{1}{8} .
\end{array}\right.
$$

Then the least favorable spectral density in the class $D_{F}$ is given by

$$
f^{\vec{\zeta}, 0}(\lambda)=\left[\frac{16}{3}\left|1-\frac{\sqrt{2}}{2} e^{-i \lambda}\right|^{2}-\left|1-\sqrt{2} e^{-i \lambda}\right|^{2}\right]_{+} .
$$

Note that $f^{\vec{\zeta}, 0}(\lambda)$ does not depend on the coefficient $\kappa$.

The minimax spectral characteristic evaluated by (16) equals

$$
h\left(f^{\vec{\zeta}, 0}, f^{\vec{\theta}}\right)=\frac{\kappa}{2}\left(1+\frac{1}{2} e^{-2 i \lambda}\right) .
$$

According to (11) the estimate of the functional $A \zeta$ is given by

$$
\hat{A} \zeta=\frac{\kappa}{2}(\zeta(0)+\theta(0))+\frac{\kappa}{4}(\zeta(-2)+\theta(-2)) .
$$

The mean square error $\hat{A} \zeta$ attains the maximal value $\Delta\left(f^{\vec{\zeta}, 0}, f^{\vec{\theta}}\right)=\frac{5}{2} \kappa^{2}$.

Example 5.2. Let $T=2, f^{\vec{\theta}}(\lambda)=I_{2}$, and $F=\left(\begin{array}{cc}2 & 6 \\ 6 & 11\end{array}\right)$. We estimate the functional

$$
A \zeta=\frac{7}{2} \zeta(1)-\sqrt{\frac{23}{2}} \zeta(0) .
$$

The least favorable spectral density is found from relation (28). In the case of the minimal rank $m=1$, the matrix coefficients $\{b(0), b(1)\}$ are solutions of conditional 
extremum problem (22)

$$
\left\{\begin{array}{c}
\left(b(0)(\sqrt{23 / 2}, \sqrt{23 / 2})^{\top}-b(1)(7 / 2,-7 / 2)^{\top}\right)\left(b^{*}(0)(\sqrt{23 / 2}, \sqrt{23 / 2})\right. \\
\left.-b^{*}(1)(7 / 2,-7 / 2)\right)+\left(b(0)(7 / 2,-7 / 2)^{\top}\right)\left(b^{*}(0)(7 / 2,-7 / 2)\right) \rightarrow \min , \\
I_{2}+b(1)\left(I_{2}+F\right) b^{*}(1)-b(0)\left(I_{2}+F\right) b^{*}(0)=\left(\begin{array}{ll}
0 & 0 \\
0 & 0
\end{array}\right) .
\end{array}\right.
$$

Then the least favorable spectral density of the class $D_{F}$ is given by

$$
f^{\vec{\zeta}, 0}(\lambda)=\left[\left(\begin{array}{cc}
2 & 6 \\
6 & 11
\end{array}\right)-\frac{178 \sqrt{23}}{10569}\left(\begin{array}{ll}
1 & 2 \\
2 & 4
\end{array}\right)\left(e^{-i \lambda / 2}+e^{i \lambda / 2}\right)\right]_{+} .
$$

The minimax spectral characteristic evaluated from formula (16) equals

$$
h\left(f^{\vec{\zeta}, 0}, f^{\vec{\theta}}\right) \approx\left(\begin{array}{c}
7.14 e^{-i \lambda}-3.23 \\
-6.74 e^{-i \lambda}-3.61
\end{array}\right) .
$$

In the calculation above we round decimals to the nearest hundredth. According to (11), the estimate of the functional $A \zeta$ is given by

$$
\begin{aligned}
\hat{A} \zeta \approx & -3.23\left(\xi_{0}(0)+\eta_{0}(0)\right)-3.61\left(\xi_{1}(0)+\eta_{1}(0)\right)+7.14\left(\xi_{0}(-1)+\eta_{0}(-1)\right) \\
& -6.74\left(\xi_{1}(-1)+\eta_{1}(-1)\right) .
\end{aligned}
$$

The maximal value of the mean square error $\hat{A} \zeta$ is $\Delta\left(f^{\vec{\zeta}, 0}, f^{\vec{\theta}}\right) \approx 23.37$.

\section{BIBLIOGRAPHY}

1. W. R. Bennett, Statistics of regenerative digital transmission, Bell Syst. Tech. 37 (1958), 15011542. MR.0102138(21:932)

2. J. Franke, On the robust prediction and interpolation of time series in the presence of correlated noise, J. Time Series Anal. 5 (1984), 227-244. MR782077 (86i:62192)

3. J. Franke, Minimax robust prediction of discrete time series, Z. Wahrsch. Verw. Gebiete 68 (1985), 337-364. MR771471 (86f:62164)

4. J. Franke and H. V. Poor, Minimax-robust filtering and finite-length robust predictors, Robust and Nonlinear Time Series Analysis, Lecture Notes in Statistics, vol. 26, Springer-Verlag, 1984. MR786305 (86i:93058)

5. E. G. Gladyshev, Periodically correlated random sequences, Doklady Akad. Nauk SSSR 137 (1961), no. 5, 1026-1029; English transl. in Soviet Math. Dokl. 2 (1961), 385-388. MR0126873 (23:A4167)

6. U. Grenander, A prediction problem in game theory, Ark. Mat. 3 (1957), 371-379. MR0090486 $(19: 822 \mathrm{~g})$

7. H. L. Hurd and A. Miamee, Periodically Correlated Random Sequences: Spectral Theory and Practice, John Wiley \& Sons, 2007. MR2348769 (2009e:62002)

8. A. N. Kolmogorov, Probability Theory and Mathematical Statistics, Collection of problems, "Nauka", Moscow, 1986; English transl., Selected works, vol. II, Mathematics and its Applications (Soviet Series), vol. 26, Kluwer Academic Publishers Group, Dordrecht, 1992; translated from the Russian by G. Lindquist; translation edited by A. N. Shiryayev. MR,1153022 (92j:01071)

9. A. Makagon, Theoretical prediction of periodically correlated sequences, Probab. Math. Statist. 19 (1999), 287-322. MR1750905 (2001m:60093)

10. A. Makagon, Stationary sequences associated with a periodically correlated sequence, Probab. Math. Statist. 31 (2011), 263-283. MR2853678 (2012j:60091)

11. M. P. Moklyachuk, Estimates of stochastic processes from observations with noise, Theory Stoch. Process. 3(19) (1997), no. 3-4, 330-338.

12. M. P. Moklyachuk, Robust procedures in time series analysis, Theory Stoch. Process. 6(22) (2000), no. 3-4, 127-147.

13. M. P. Moklyachuk, Game theory and convex optimization methods in robust estimation problems, Theory Stoch. Process. 7(23) (2001), no. 1-2, 253-264.

14. M. P. Moklyachuk, Robust Estimates for Functionals of Stochastic Processes, "Kyivs'kyi Universytet", Kyiv, 2008. (Ukrainian) 
15. M. P. Moklyachuk and O. Yu. Masyutka, On the problem of filtration for stationary vector sequences, Teor. Imovir. Matem. Statyst. 75 (2007), 95-104; English transl. in Theor. Probability Math. Statist. 75 (2007), 109-119. MR2321185

16. B. N. Pshenichny , Necessary Conditions for an Extremum, "Nauka", Moscow, 1982; English transl., Marcel Dekker, Inc., New York, 1971. MR686452 (84c:49003)

17. Yu. A. Rozanov, Stationary Random Processes, 2nd ed., "Nauka", Moscow, 1990. (Russian) MR:1090826 (92d:60046)

18. N. Wiener, Extrapolation, Interpolation and Smoothing of Stationary Time Series. With Engineering Applications, The M. I. T. Press, Massachusetts Institute of Technology, Cambridge, Mass., 1966. MR0031213(11:118j)

19. A. M. Yaglom, Correlation Theory of Stationary and Related Random Functions. Vol. 1: Basic Results, Springer Series in Statistics, Springer-Verlag, New York, etc., 1987. MR893393 (89a:60105)

20. A. M. Yaglom, Correlation Theory of Stationary and Related Random Functions. Vol. 2: Supplementary Notes and References, Springer Series in Statistics, Springer-Verlag, New York, etc., 1987. MR915557 (89a:60106)

Department of Probability Theory, Statistics, and Actuarial Mathematics, Faculty for Mechanics and Mathematics, National Taras Shevchenko University, Academician Glushkov Avenue, 4-E, Kiev 03127, Ukraine

E-mail address: idubovetska@gmail.com

Department of Probability Theory, Statistics, and Actuarial Mathematics, Faculty for Mechanics and Mathematics, National Taras Shevchenko University, Academician Glushkov Avenue, 4-E, Kiev 03127, Ukraine

E-mail address: mmp@univ.kiev.ua

Received 21/NOV/2011

Translated by N. SEMENOV 